TRANSACTIONS OF THE

AMERICAN MATHEMATICAL SOCIETY

Volume 177, March 1973

\title{
KRULL DIMENSION IN POWER SERIES RINGS
}

BY

JIMMY T. ARNOLD

ABSTRACT. Let $R$ denote a commutative ring with identity. If there exists a chain $P_{0} \subset P_{1} \subset \ldots \subset P_{n}$ of $n+1$ prime ideals of $R$, where $P_{n} \neq R$, but no such chain of $n+2$ prime ideals, then we say that $R$ has dimension $n$. The power series ring $R[[X]]$ may have infinite dimension even though $R$ has finite dimension.

1. Introduction. We shall write $\operatorname{dim} R=n$ to denote that $R$ has dimension $n$. Seidenberg, in [6] and [7], has investigated the the ory of dimension in rings of polynomials. In particular, he has shown in [6] that if $\operatorname{dim} R=n$, then $n+1 \leq$ $\operatorname{dim} R[X] \leq 2 n+1$, where $X$ is an indeterminate over $R$. One might now ask whether it is also true that $n+1 \leq \operatorname{dim} R[[X]] \leq 2 n+1$. It is easy to show that $n+1 \leq \operatorname{dim} R[[X]]$ when $\operatorname{dim} R=n$. In [3] Fields has considered the theory of dimension in power series rings over valuation rings. Using results obtained by Fields, Arnold and Brewer have noted in $[1]$ that $\operatorname{dim} V[[X]] \geq 4$ for any rank one nondiscrete valuation ring $V$. Thus, if $\operatorname{dim} R=n$, then $2 n+1$ is not, in general, an upper bound for $\operatorname{dim} R[[X]]$. In this paper we show that we may have $\operatorname{dim} R[[X]]=\infty$ even though $R$ has finite dimension. Our main result is Theorem 1 , which gives sufficient conditions on a ring $R$ in order that $\operatorname{dim} R[[X]]=\infty$. In fact, the conditions given insure the existence of an infinite ascending chain of prime ideals in $R[[X]]$.

Throughout this paper, $R$ denotes a commutative ring with identity, $\omega$ is the set of natural numbers, and $\omega_{0}$ is the set of nonnegative integers. If $f(X)=$ $\sum_{i=0}^{\infty} a_{i} X^{i} \in R[[X]]$, then we denote by $A_{f}$ the ideal of $R$ generated by the coefficients of $f(X)$. For an ideal $A$ of $R$, we let $A[[X]]=\left\{f(x)=\sum_{i=0}^{\infty} a_{i} X^{i} \mid a_{i} \in A\right.$ for each $\left.i \in \omega_{0}\right\}$ and we define $A R[[X]]$ to be the ideal of $R[[X]]$ which is generated by $A$. Thus, $A R[[X]]=\{f(X) \mid A, \subseteq B$ for some finitely generated ideal $B$ of $R$, with $B \subseteq A\}$. We shall say that the ideal $A$ is an ideal of strong finite type (or an SFT-ideal) provided there is a finitely generated ideal $B \subseteq A$ and $k \in \omega$ such that $a^{k} \in B$ for each $a \in A$. If each ideal of $R$ is an SFT-ideal, then we say that $R$ satisfies the SFT-property. Throughout, our notation and terminology are essentially that of [4].

Presented to the Society, January 21, 1971; received by the editors February 21, 1972. AMS (MOS) subject classifications (1969). Primary 1393; Secondary 1320.

Key words and phrases. Dimension, power series ring.

Copyright ๑ 1973, American Mathematical Society 
2. Main Theorem. Let $R$ be a ring which does not satisfy the SFT-property. If $M$ is an ideal of $R$ which is not an SFT-ideal, then we may choose a sequence $\left\{a_{i}\right\}_{i=0}^{\infty}$ of elements of $M$ so that $a_{k+1}^{k+1} \notin\left(a_{0}, \cdots, a_{k}\right)$ for each $k \in \omega_{0}$. Set $A_{k}=$ $\left(a_{0}, \cdots, a_{k}\right)$ and let $A=\bigcup_{k=0}^{\infty} A_{k}$. For each $m \in \omega$, we now choose a sequence $\left\{a_{m, i}\right\}_{i=0}^{\infty}$ of elements of $A$ as follows. For $m=1$, we take $a_{1, i}=a_{i}$ for each $i \in \omega_{0}$. Having defined the sequence $\left\{a_{m, i}\right\}_{i=0}^{\infty}$ for $1 \leq m<n$, we define the sequence $\left\{a_{n, i}\right\}_{i=0}^{\infty}$ by taking $a_{n, i}=a_{n-1, i}{ }^{2+1}$ for each $i \in \omega_{0}$. For each $n \in \omega$ we set $f_{(n)}=\sum_{i=0}^{\infty} a_{n, i} X^{i}$.

Definition 1. Suppose that $g(X) \in R[[X]], g(X)=\Sigma b_{i} X^{i}$, and let $n, m, \mu, r$ be integers such that $m \geq n \geq 1$, and $r \geq 0$. We shall say that the tuple $(g, m, \mu, r)$ has property $(n)$ if for $i \geq r$ there exists an integer $t_{i}$ such that the following hold, where we assume that $a_{m, i}=a_{n, k_{i}}=a_{1, s_{i}}$.

(i) $b_{t_{i}}=a_{m, i}^{\mu}+\alpha$ for some $a \in \dot{A}_{s_{i}-1}$.

(ii) $t_{i} \leq \mu k_{i}$.

(iii) $b_{j} \in A_{s_{i-1}}$ for $0 \leq j<t_{i}$.

For $n \in \omega$, we set $S_{n}=\{g(X) \in R[[X]] \mid(g, m, \mu, r)$ has property $(n)$ for some $\mathrm{m}, \mu \in \omega$ and $\left.r \in \omega_{0}\right\} . S_{n}$ is nonempty since $\left(f_{(n)}, n, 1,0\right)$ satisfies property $(n)$.

Lemma 1. If $n, n_{1} \in \omega$ are such that $n \geq n_{1}$, then $S_{n} \subseteq S_{n_{1}}$.

Proof. Suppose that $g(X) \in S_{n}$ and that $(g, m, \mu, r)$ has property $(n)$. We wish to see that $(g, m, \mu, r)$ also has property $\left(n_{1}\right)$. But properties (i) and (iii) of Definition 1 already hold since they are independent of the choice of $n$. To see that (ii) holds, suppose that $i \geq r$ and that $a_{m, i}=a_{n, k_{i}}=a_{n_{1}, v_{i}}$. Then $k_{i} \leq v_{i}$, and hence $t_{i} \leq \mu k_{i} \leq \mu v_{i}$. It follows that $g(X) \in S_{n_{1}}$.

Lemma 2. For each $n \in \omega, S_{n}$ is a multiplicatively closed subset of $R[[X]]$.

Proof. Let $g(X) \in R[[X]], g(X)=\sum_{i=0}^{\infty} b_{i} X^{i}$. We first show that if $(g, m, \mu, r)$ has property $(n)$ and if $m_{1} \geq m$, then $\left(g, m_{1}, \mu, r\right)$ also has property $(n)$. Thus, suppose that $i \geq r$ and that $a_{m_{1, i}}=a_{m, j_{i}}=a_{n, k_{i}}=a_{1, s_{i}}$. Since $j_{i} \geq i \geq r$, there exists an integer $t_{j_{i}}$ such that:

(i) $b_{t_{j}}=a_{m, j_{i}}^{\mu}+\alpha$ for some $\alpha \in A_{s_{i}-1}$.

(ii) $t_{j_{i}} \leq \mu k_{i}$.

(iii) $b_{\lambda} \in A_{s_{i-1}}$ for $0 \leq \lambda<t_{j_{i}}$.

Taking $\tau_{i}=t_{j_{i}}$ and using the fact that $a_{m_{1}, i}=a_{m, j_{i}}$, we see that $\tau_{i}$ satisfies properties (i), (ii) and (iii) of Definition 1 so $\left(g, m_{1}, \mu, r\right)$ has property $(n)$.

Now let $g(X), b(X) \in S_{n}$, where $g(X)=\sum_{i=0}^{\infty} b_{i} X^{i}$ and $b(X)=\sum_{i=0}^{\infty} c_{i} X^{i}$, and suppose that $\left(g, m_{1}, \mu_{1}, r_{1}\right)$ and $\left(b, m_{2}, \mu_{2}, r_{2}\right)$ satisfy property $(n)$. By the preceding remarks, we may assume that $m_{1}=m_{2}$ and, clearly, we may assume that $r_{1}=r_{2}$. Set $m=m_{1}=m_{2}$ and $r=r_{1}=r_{2}$. We wish to show that $\left(g h, m, \mu_{1}+\mu_{2}, r\right)$ 
has property $(n)$. Suppose that $i \geq r$ and that $a_{m, i}=a_{n, k_{i}}=a_{1, s_{i}}$. By assumption there exist integers $t_{i}$ and $\tau_{i}$ such that $b_{t_{i}}=a_{m, i}^{\mu_{1}}+\alpha$ and $c_{\tau_{i}}=a_{m, i}^{\mu_{2}}+\beta$ for some $\alpha, \beta \in A_{s_{i-1}}$. Moreover, $b_{\lambda}, c_{\delta} \in A_{s_{i-1}}$ for $0 \leq \lambda<t_{i}$ and $0 \leq \delta<\tau_{i}$. If $g(X) b(X)=\sum_{j=0}^{\infty} \xi_{j} X^{j}$, then

$$
\xi_{t_{i}+\tau_{i}}=b_{t_{i}} c_{\tau_{i}}+\sum_{\lambda+\delta=t_{i}+\tau_{i} ; \lambda \neq t_{i} ; \delta \neq \tau_{i}} b_{\lambda} c_{\delta} .
$$

But if $\lambda \neq t_{i}$ and $\delta \neq \tau_{i}$, then either $\lambda<t_{i}$ or $\delta<\tau_{i}$. Consequently, either $b_{\lambda} \in A_{s_{i}-1}$ or $c_{\delta} \in A_{s_{i}-1}$. Since $b_{t_{i}} c_{\tau_{i}}=a \mu_{m, i}^{\mu_{1}+\mu_{2}}+\alpha a_{m, i}^{\mu_{2}}+\beta a_{m, i}^{\mu_{1}}+\alpha \beta$, it follows that $\xi_{t_{i}+\tau_{i}}=a_{m, i}^{\mu_{1}+\mu_{2}}+\gamma$ for some $\gamma \in A_{s_{i}-1}$. By assumption, we have $t_{i} \leq \mu_{1} k_{i}$ and $\tau_{i} \leq \mu_{2} k_{i}$. Therefore, $t_{i}+\tau_{i} \leq\left(\mu_{1}+\mu_{2}\right) k_{i}$. Finally, if $0 \leq \lambda<t_{i}+\tau_{i}$, then $\xi_{\lambda}=$ $\sum_{j=0}^{\lambda} b_{j} c_{\lambda-j} \in A_{s_{i}-1}$ since either $j<t_{i}$ or $\lambda-j<\tau_{i}$.

Lemma 3. Let $n, \nu \in \omega$ be such that $n>\nu$. If $g(X) \in S_{n}$, then $g(X)+$ $b(X) f_{(\nu)}(X) \in S_{n}$ for arbitrary $b(X) \in R[[X]]$.

Proof. Suppose that $g(X)=\sum_{i=0}^{\infty} b_{i} X^{i}$ and that $(g, m, \mu, r)$ has property $(n)$. Let $\eta=\min \left\{i \in \omega_{0} \mid a_{m, i}=a_{n, k_{i}} \Rightarrow k_{i} \geq \mu\right\}$ and set $r_{1}=\max \{r, \eta\}$. If $q(X)=$ $g(X)+b(X) f_{(\nu)}(X)=\sum_{i=0}^{\infty} \xi_{i} X^{i}$, then we wish to show that $\left(q, m, \mu, r_{1}\right)$ satisfies roperty $(n)$. Thus, suppose that $i \geq r_{1}$ and that $a_{m, i}=a_{n, k_{i}}=a_{\nu, \lambda_{i}}=a_{1, s_{i}}$. By issumption, there exists an integer $t_{i}$ such that $b_{t_{i}}=a_{m, i}^{\mu}+\alpha$ for some $\alpha \epsilon$ $A_{s_{i}-1}$ and such that $t_{i} \leq \mu k_{i} \leq k_{i}^{2}$. Since $\lambda_{i} \geq k_{i}^{2}+1$, it follows that $a_{\nu, j} \in A_{s_{i-1}}$ for $0 \leq j \leq t_{i}$. Consequently, if $b(X)=\sum_{j=0}^{\infty} c_{j} X^{j}$ and $b(X) f_{(\nu)}(X)=\sum_{i=0}^{\infty} \gamma_{j} X^{j}$, then $\gamma_{t_{i}}=\Sigma_{j=0}^{t_{i}} a_{\nu, j} c_{t_{i}-j} \in A_{s_{i}-1}$. Therefore, $\xi_{t_{i}}=b_{t_{i}}+\gamma_{t_{i}}=a_{m, i}^{\mu}+\alpha+\gamma_{t_{i}}$ and (i) of Definition 1 is satisfied. We already have that $t_{i} \leq \mu k_{i}$, so (ii) is also satisfied. To see that (iii) holds, suppose that $0 \leq \delta<t_{i}$. By assumption, we have that $b_{\delta} \in A_{s_{i}-1}$. Also, $\gamma_{\delta}=\Sigma_{j=0}^{\delta} a_{\nu, j} c_{t_{i}-j} \in A_{s_{i}-1}$, since $j \leq \delta<t_{i} \leq k_{i}^{2}$ implies that $a_{\nu, j} \in A_{s_{i}-1}$. Consequently, $\xi_{\delta}=b_{\delta}+\gamma_{\delta} \in A_{s_{i-1}}$ and our proof is complete.

We now state our main result.

Theorem 1. Let $R$ be a commutative ring with identity. The following conditions are equivalent and imply that $R[[X]]$ bas infinite dimension.

(1) $R$ does not satisfy the SFT-property.

(2) There exists an ideal $A$ of $R$ such that $A[[X]] \not \subset \sqrt{A R[[X]]}$.

(3) There exists a prime ideal $P$ of $R$ such that $P[[X]] \neq \sqrt{P R[[X\rceil]}$.

Proof. Assume that (1) holds. We shall $\mathrm{f}$ irst prove that $\operatorname{dim} R[[X]]=\infty$. Let the ideal $A$ be as previously defined. We wish to see that $A R[[X]] \cap S_{1}=\varnothing$. Thus, let $g(X) \in A R[[X]] \cap S_{1}, g(X)=\sum_{i=0}^{\infty} b_{i} X^{i}$. Then $A_{g} \subseteq C$ for some finitely generated ideal $C$ of $R$, where $C \subseteq A$. Consequently, there exists $k \epsilon \omega_{0}$ such that $A_{g} \subseteq A_{k}$. Suppose that $(g, m, \mu, r)$ has property (1) and that $r$ has been chosen so that if $i \geq r$ and $a_{m, i}=a_{1, s_{i}}$, then $s_{i}>\max \{\mu, k\}$. If $t_{i}$ is such that $b_{t_{i}}=a_{m, i}^{\mu}+\alpha$ for some $\alpha \in A_{s_{i-1}}$, then we have $a_{m, i}^{\mu}+\alpha \in A_{k} \subseteq A_{s_{i-1}}$. 
Therefore, $a_{m, i}^{\mu} \in A_{s_{i}-1}$, a contradiction since $\mathrm{a}_{m, i}^{s_{i}}=a_{i, s_{i}}^{s_{i}} \notin A_{s_{i}-1}$ and $s_{i}>\mu$. (Since $f_{(1)} \in S_{1}$, it follows that $f_{(1)} \in A[[X]]-\sqrt{A R[[X]]}$. Thus we see that (1) implies (2).) But $S_{1} \cap A R[[X]]=\varnothing$ implies the existence of a prime ideal $P_{1}$ of $R[[X]]$ such that $A R[[X]] \subseteq P_{1}$ and $P_{1} \cap S_{1}=\varnothing$. Suppose there exists a chain $P_{1} \subset \ldots \subset P_{n}$ of prime ideals of $R[[X]]$ such that $P_{n} \cap S_{n}=\varnothing$, and let $C_{n}=$ $P_{n}+\left(f_{(n)}(X)\right)$. If $g(X) \in S_{n+1}$, then by Lemma $3, g(X)+b(X) f_{(n)}(X) \in S_{n+1} \subseteq S_{n}$ for arbitrary $b(X) \in R[[X]]$. It follows that $g(X)+b(X) f_{(n)}(X) \notin P_{n}$ and hence that $g(X) \notin C_{n}$. Thus, $C_{n} \cap S_{n+1}=\varnothing$ and there exists a prime ideal $P_{n+1}$ such that $P_{n} \subset C_{n} \subseteq P_{n+1}$ and $P_{n+1} \cap S_{n+1}=\varnothing$. We see by induction that $\operatorname{dim} R[[X]]_{x}$.

To see that (2) implies (3), we note that if $A[[X]] \not \subset \sqrt{A R[[X]}]$, then there exists a prime ideal $Q$ of $R[[X]]$ such that $A R[[X]] \subseteq Q$ but $A[[X]] \subseteq \subseteq Q$. If $P=Q \cap R$, then $P \supseteq A$ and hence $P[[X]] \supseteq A[[X]]$. Therefore, $Q \supseteq P R[[X]]$ but $Q \underline{\not} P[[X]]$. It follows that $P[[X]] \neq \sqrt{P R[[X]]}$. In order to show that $(3)$ implies (1), we require the following lemma.

Lemma 4. Let $A$ be an ideal of $R$ and suppose that there exists $k \in \omega$ sucb that $a^{k}=0$ for each $a \in A$. If $f(X) \in A[[X]]$, then $f(X)$ is nilpotent.

Proof. We first prove the existence of an integer $m$ such that $m \xi=0$ for all $\xi \in A^{m}$. Suppose we have integers $\mu, \nu_{1}, \cdots, \nu_{t}$ such that $\mu a_{1}^{\nu_{1}} \cdots a_{t}^{\nu_{t}}=0$ for all $a_{1}, \cdots, a_{t} \in A$ (certainly this condition is satisfied if $\mu=t=1$ and $\nu_{1}=k$ ) and suppose that $\nu_{i} \geq 2$ for some $i, 1 \leq i \leq t$. For convenience, we suppose that $\nu_{1} \geq 2$. Now let $b_{0}, b_{1}, \cdots, b_{t} \in A$. By assumption, we have that

$$
0=\mu\left(b_{0}+b_{1}\right)^{\nu_{1}} b_{2}^{\nu_{2}} \cdots b_{t}^{\nu_{t}}=\mu b_{0}^{\nu_{1}-2}\left(b_{0}+b_{1}\right)^{\nu_{1}} b_{2}^{\nu_{2}} \cdots b_{t}^{\nu_{t}}=\sum_{j=0}^{\nu_{1}} \xi_{j},
$$

where $\xi_{j}=\mu\left({ }^{\nu_{1}}\right) b_{0}^{2 \nu_{1}-j-2} b_{1}^{j} b_{2}^{\nu_{2}} \ldots b_{t}^{\nu_{t}}$. If $0 \leq j \leq \nu_{1}-2$, then $2 \nu_{1}-j-2 \geq \nu_{1}$ so that $\xi_{j}=0$. Also, $\xi_{\nu_{1}}=b_{0}^{\nu_{1}-2}\left(\mu b_{1}^{\nu_{1}} \ldots b_{t}^{\nu_{t}}\right)=0$. It follows that $0=\xi_{\nu_{1}-1}=$ $\mu \nu_{1} b_{0}^{\nu_{1}-1} b_{1}^{\nu_{1}-1} b_{2}^{\nu_{2}} \cdots b_{t}^{\nu_{t}}$. By a finite number of repetitions of this procedure, we may find integers $\mu$ and $t$ such that $\mu a_{1} \cdots a_{t}=0$ for all $a_{1}, \cdots, a_{t} \in A$. If we set $m=\mu t$, then $m A^{m}=(0)$. Now let $f(X) \in A[[X]], f(X)=\sum_{i=0}^{\infty} a_{i} X^{i}$. Follow ing a proof given by Fields [2, Theorem 1] we suppose that $m=p$ is a prime integer. Then $(f(X))^{p^{k}}=\sum_{i=0}^{\infty} a_{i}^{p^{k}} X^{i p^{k}}=0$. If $m$ is not prime and $m=p_{1}^{e_{1}} \cdots p_{t}^{e_{t}}$ is a prime factorization for $m$, then let $\phi_{j}: R[[X]] \rightarrow\left(R / p_{j} A^{p_{j}}\right)[[X]]$ be the canonical homomor phism for $1 \leq j \leq t$. By the previous case for $m$ a prime, we have $0=\left[\phi_{j}(f(X))\right]^{p_{j}^{k}}$, that is $(f(X))^{p_{j}^{k}} \in p_{j} A^{p_{j}}[[X]]$. If $n=\left(p_{1}^{e_{1}{ }^{k}}+\cdots+p_{t}^{e_{t}{ }^{k}}\right)_{m}$, then

$$
\begin{aligned}
& (f(X))^{n}-\left[\left((f(X))^{\left.p_{1}^{k}\right)^{e}}{ }^{e} \ldots\left((f(X))^{p_{t}^{k}}\right)^{e} t\right]^{m} \in\left[\left(p_{1} A^{p_{1}}\right)^{e_{1}}[[X]] \ldots\left(p_{t} A^{p_{t}}\right)^{e_{t}}[[X]]\right]^{m}\right. \\
& \subseteq m A^{m}[[X]]=(0) \text {. }
\end{aligned}
$$


To complete the proof of Theorem 1, suppose that $B$ is an ideal of $R$ which is an SFT-ideal. By definition, there exists $k \in \omega$ and a finitely generated ideal $C \subseteq B$ such that $b^{k} \in C$ for all $b \in B$. Setting $\bar{R}=R / C$ and $\bar{B}=B / C$, it follows from Lemma 4 that $f(X)$ is nilpotent for each $f(X) \in \bar{B}[[X]]$. Therefore, if $g(X) \epsilon$ $B[[X]]$, then $g(X) \in \sqrt{C[[X]]}=\sqrt{C R[[X]]} \subseteq \sqrt{B R[[X]]}$. Consequently, if $P$ is a prime ideal of $R$ such that $P[[X]] \not \sqrt{P R[[X]]}$, then $P$ is not an SFT-ideal. This proves that (3) implies (1) and the theorem follows.

If $\operatorname{dim} R=n$, then it is natural to ask whether the conditions given in Theorem 1 are necessary in order that $\operatorname{dim} R[[X]]=\infty$. Another interesting question which arises is whether the following conditions are equivalent:

(1) $\operatorname{dim} R[[X]] \neq n+1$.

(2) $\operatorname{dim} R[[X]]=\infty$.

We show that both these questions can be answered affirmatively if $\operatorname{dim} R=0$.

Theorem 2. Let $R$ be a commutative ring with identity and suppose that $\operatorname{dim} R=0$. Then the following statements are equivalent:

(1) $\operatorname{dim} R[[X]] \neq 1$.

(2) $\operatorname{dim} R[[X]]=\infty$.

(3) $R$ contains a maximal ideal $M$ such that $M[[X]] \neq \sqrt{M R[[X]]}$.

Proof. We have already seen that (3) implies (2) and clearly, (2) implies (1). Suppose that (1) holds and let $Q_{0} \subset Q_{\mathbf{p}} \subset Q_{2} \subset R[[X]]$ be a chain of prime ideals of $R[[X]]$. If $M=Q_{0} \cap R$, then $M$ is a maximal ideal of $R$ so we have $M=Q_{0} \cap$ $R=Q_{1} \cap R=Q_{2} \cap R$. Now $Q_{0} \underline{\not} M[[X]]$ since $R[[X]] / M[[X]] \cong(R / M)[[X]]$ is a rank one discrete valuation ring. But by $[1$, Proposition 1$]$, either $Q_{0} \subset M[[X]]$ or $Q_{0} \supseteq M[[X]]$. Therefore, $M R[[X]] \subseteq Q_{0} \subset M[[X]]$ and $M[[X]] \neq \sqrt{M R[[X]]}$.

3. Examples. We conclude by providing three examples of finite dimensional rings $R$ such that $\operatorname{dim} R[[X]]=\infty$.

Example 1. If $V$ is a rank one nondiscrete valuation ring, then $\operatorname{dim} V[[X]]=\infty$. More generally, if $V$ is a valuation ring which contains an idempotent prime ideal $P$, then $P$ is not an SFT-ideal.so $\operatorname{dim} V[[X]]=\infty$.

Example 2. An integral domain $D$ is said to be almost Dedekind provided $D_{M}$ is a Noetherian valuation ring for each maximal ideal $M$ of $D$. Let $D$ be any almost Dedekind domain which is not Dedekind [4, p. 586], and let $M$ be a maximal ideal of $D$ which is not finitely generated. It follows from Theorem 29.4 of $[4$, p. 411$]$ that $M$ is not the radical of a finitely generated ideal. Thus, $M$ is not an SFT-ideal and $\operatorname{dim} D[[X]]=\infty$. More generally, if $R$ is a commutative ring with identity which does not have Noetherian prime spectrum, then $\operatorname{dim} R[[X]]=\infty$. This is an immediate consequence of Corollary 2.4 of [5] which states that a ring $R$ has Noetherian prime spectrum if and only if each prime ideal of $R$ is the radical of a finitely generated ideal. Example 1 and the following example illustrate 
that we may have $\operatorname{dim} R[[X]]=\infty$ even though $R$ has Noetherian prime spectrum.

Example 3. Let $\left\{Y_{i}\right\}_{i=0}^{\infty}$ be a collection of indeterminates over $Q$, the field of rationals, and set $R=Q\left[Y_{0}, Y_{1}, \ldots\right] /\left(Y_{0}^{n}, Y_{1}^{n}, \ldots\right)$, where $n$ is a positive integer and $n \geq 2$. We note that $\operatorname{dim} R=0$ and that $M=\left(\bar{Y}_{0}, \bar{Y}_{1}, \ldots\right)$ is the unique proper prime ideal of $R$. If $f(X)=\sum_{i=0}^{\infty} \bar{Y}_{i} X^{i}$, then Fields proves in [2] that $f(X)$ is not nilpotent. If $g(X) \in M R[[X]]$, then $g(X)=\sum_{i=0}^{\bar{t}} \bar{Y}_{i} b_{i}(X)$ for some $t \in \omega$ and $b_{i}(X) \in R[[X]]$. Since $\bar{Y}_{i}^{n}=0$ for $0 \leq i \leq t$, it follows that $g(X)$ is nilpotent. Consequently, $f(X) \notin \sqrt{M R[[X]]}$ so, by Theorem $1, \operatorname{dim} R[[X]]=\infty$.

\section{BIBLIOGRAPHY}

1. J. T. Arnold and J. W. Brewer, When $(D[[X]]))_{P}[[X]]$ is a valuation ring, Proc. Amer. Math. Soc. 37 (1973), 326-332.

2. D. E. Fields, Zero divisors and nilpotent elements in power series rings, Proc. Amer. Math. Soc. 27 (1971), 427-433. MR 42 \#5983.

3. —_ Dimension theory in power series rings, Pacific J. Math. 35 (1970), 601611. MR $43 \# 3251$.

4. R. Gilmer, Multiplicative ideal the ory, Queen's Papers in Pure and Appl. Math., no. 12, Queen's University, Kingston, Ont., 1968. MR 37 \#5198.

5. J. Ohm and R. Pendleton, Rings with Noetherian spectrum, Duke Math J. 35 (1968), 631-639. MR 37 \#5201.

6. A. Seidenberg, A note on the dimension theory of rings, Pacific J. Math. 3 (1953), 505-512. MR 14, 941.

7. - On the dimension theory of rings. II, Pacific J. Math. 4 (1954), 603-614. MR 16, 441.

DEPARTMENT OF MATHEMATICS, VIRGINIA POLYTECHNIC INSTITUTE AND STATE UNIVERSITY, BLACKSBURG, VIRGINIA 24061 